\section{Response to "Potential Misclassification of Urinary Tract Related Bacteremia Upon Applying the 2015 Catheter-Associated Urinary Tract Infection Surveillance Definition From the National Healthcare Safety Network"}

To The Editor-In their concise communication, "Potential Misclassification of Urinary Tract Related Bacteremia Upon Applying the 2015 Catheter-Associated Urinary Tract Infection Surveillance Definition From the National Healthcare Safety Network," "Greene et al present findings from their retrospective review of cases they define as urinary tract-related bloodstream infection at 3 VA hospitals. The authors report that among 145 cases with documented indwelling urinary catheters, 93 cases $(64.1 \%)$ would be deemed catheter-associated urinary tract infections (CAUTIs) according to the updated 2015 National Healthcare Safety Network (NHSN) criteria. The authors conclude that applying those criteria, specifically the criterion introduced in 2015 that requires a urine culture bacterial count of at least $1 \times 10^{5}$ colony-forming units $(\mathrm{CFU} / \mathrm{mL})$, would lead to under-ascertainment of clinically meaningful CAUTIs.

The authors' concern that "the new CDC surveillance definition has the potential to underestimate the burden of CAUTIrelated illness" and that "this has the potential to undermine the faith that clinicians have in the reliability of the national surveillance system for CAUTI," must be balanced by what was previously an even larger "disconnect" and widely shared concerns about a lack of clinical credibility. A study comparing clinical CAUTI determinations to earlier NHSN definitions indicated that the NHSN definitions had a positive predictive value of only 35\% compared to Infectious Diseases consultant evaluation. ${ }^{2}$ While the CAUTI surveillance criteria that the CDC introduced in 2015 may omit some infections that are deemed clinically significant, the magnitude of missed cases associated with bloodstream infections likely is small, and this possible shortcoming should be placed in the larger context of criteria changes that improve the specificity of case findings. An analysis of NHSN data, completed as part of the review of predecessor CAUTI criteria, showed that the $\mathrm{CFU} / \mathrm{mL}$ threshold change would result in $10 \%$ fewer reported CAUTIs; only $0.5 \%$ of all CAUTIs with a reported secondary bloodstream infection would not be reported as a result of the change, much lower than that identified by Green et al. ${ }^{3}$ Furthermore, many of the "secondary bloodstream infections" previously attributed to the urinary tract may still (and perhaps more appropriately) be captured in NHSN surveillance as central-line-associated bloodstream infections.

The authors suggest "moving toward a clinician-based approach for diagnosis" as an alternative to the current NHSN criteria. Clinical and surveillance definitions serve different purposes.
Clinical definitions of UTI are used by clinicians for individual patient diagnosis, involve clinical judgment and consideration of all relevant clinical data, and guide treatment decisions. In contrast, surveillance definitions are aimed at populations, with the purposes of measuring disease incidence and outcomes and evaluating the impact of prevention activities. To be applicable and informative in multiple settings, surveillance definitions must be based on a circumscribed set of data. Because these definitions are used for performance measurement, the CDC is obligated to select laboratory tests and test result criteria that can be applied as widely as possible. A recent survey by the Association for Professionals in Infection Control and Epidemiology provides evidence of the variability of laboratory standards for working up and reporting urine specimens. ${ }^{3}$ Until a standard clinical definition is available that can be operationalized for use in surveillance and widely adopted, the NHSN must continue to provide CAUTI criteria that enable intra- and inter-facility comparisons, and serve as a meaningful catalyst for infection prevention in US healthcare facilities.

\section{ACKNOWLEDGMENTS}

Financial support: No financial support was provided relevant to this article.

Potential conflicts of interest: All authors report no conflicts of interest relevant to this article.

\section{Katherine Allen-Bridson, BSN, MScPH, CIC;} Daniel Pollock, MD

Affiliations: Centers for Disease Control and Prevention, National Center for Emerging and Zoonotic Infectious Disease, Division of Healthcare Quality Promotion, Atlanta, Georgia.

Address correspondence to Katherine Allen-Bridson, 1600 Clifton Rd MS A-24, Atlanta, GA Fsa6@cdc.gov.

Infect Control Hosp Epidemiol 2016;37:1121-1121

(C) 2016 by The Society for Healthcare Epidemiology of America. All rights reserved. 0899-823X/2016/3709-0021. DOI: 10.1017/ice.2016.142

\section{REFERENCES}

1. Greene MT, Ratz D, Meddings J, Fakih MG, Saint S. Potential misclassification of urinary tract-related bacteremia upon applying the 2015 catheter-associated urinary tract infection surveillance definition from the National Healthcare Safety Network. Infect Control Hosp Epidemiol, available on CJO2016. doi:10.1017/ice.2015.339.

2. Hanna FA, Sambirska O, Iyer S, Szpunar S, Fakih MG. Clinician practice and the National Healthcare Safety Network definition for the diagnosis of catheter-associated urinary tract infection. Am J Infect Control 2013;41:1173-1177.

3. Allen-Bridson K, Pollock D, Gould CV. Promoting prevention through meaningful measures: improving the Centers for Disease Control and Prevention's National Healthcare Safety Network urinary tract infection surveillance definitions. Am J Infect Control 2015;43:1096-1098. 


\section{Response to Allen-Bridson and Pollock}

To The Editor-We appreciate the opportunity to respond to Allen-Bridson and Pollock and thank them for their thoughtful letter. We value the data provided in their letter, which helps to illustrate that the overall under-ascertainment of clinically meaningful catheter-associated urinary tract infection (CAUTI) is likely to be lower than we reported in our study, which was purposively constrained to urinary tractrelated bloodstream infection. We also recognize that surveillance and clinical definitions are used for different purposes and may not always align.

We would, however, like to raise the following salient points. First, although the Centers for Disease Control and Prevention (CDC) National Health Safety Network (NHSN) CAUTI measures are surveillance based, they are widely used for quality improvement assessment, are tied to compensation, and are often used interchangeably as clinical CAUTI events deemed "potentially preventable." Thus, it is important for the surveillance measures to be considered clinically relevant.

Second, using historical data containing CAUTIs reported under previous definitions, the CDC reported that the recent change in the CAUTI definition led to a drop in the CAUTI standardized infection ratio to 0.55 , with a corresponding reduction in number of attributable events. ${ }^{1}$ Many hospitals might mistakenly view this reduction based purely on definitional change as improvement, thereby no longer focusing on inappropriate urinary catheter use. Additionally, the capture of data regarding previously attributed bacteremic CAUTIs through reclassification as central-line bloodstream infection (CLABSIs) denotes a shift in diagnosis and not necessarily an improved diagnosis of CLABSI. The NHSN CLABSI definition is one of exclusion; thus, bacteremia with no other primary source defined by NHSN often ends up being labeled CLABSI. Attributing bacteremia due to urinary tract infection as CLABSI may pose issues because the preventive and therapeutic measures for CAUTI and CLABSI differ.

Third, we agree that with a clinician-based approach, it is difficult to objectively measure for accuracy of diagnosis. However, patients labeled by clinicians as having CAUTI that receive antimicrobials represent a population that includes symptomatic CAUTI and asymptomatic bacteriuria. These are both relevant safety issues for patients in the hospital setting: one is related to device harm and the other is associated with unnecessary antimicrobial harm.

Finally, our conclusion was to consider alternative modifications to the CAUTI surveillance definition; clinician-based diagnosis was provided as one possible example. We further advocate the use of the device utilization ratio as an objective measure that reflects all potential risks (infectious and non-infectious) associated with the urinary catheter. ${ }^{2}$

We applaud the CDC for their efforts in enhancing patient safety. Although we are not promoting clinician-based diagnosis of CAUTI as the panacea, we believe that taking it and other alternatives into consideration have a place in modifying and improving the CAUTI definition. While this process will require several iterations before deriving a surveillance definition that best represents the clinical practice of CAUTI prevention and care, it will be a worthwhile pursuit because it will provide an incredibly powerful tool that can be used to both prevent CAUTI and reduce inappropriate antimicrobial use.

\section{ACKNOWLEDGMENTS}

Financial support: This work was supported by a Veterans Affairs Clinical Sciences Research \& Development Merit Review Award (grant no. EPID-011-11S).

Potential conflicts of interest: The authors report no conflicts of interest in relation to this letter.

\section{Todd Greene, $\mathrm{PhD}, \mathrm{MPH} ;{ }^{1,2,3}$ Mohamad G. Fakih, MD, MPH; Sanjay Saint, MD, MPH ${ }^{1,2,3}$}

Affiliations: 1. Department of Internal Medicine, University of Michigan Medical School, Ann Arbor, Michigan; 2. VA/UM Patient Safety Enhancement Program, Ann Arbor, Michigan; 3. VA Ann Arbor Healthcare System, Ann Arbor, Michigan; 4. St. John Hospital and Medical Center, Detroit, Michigan.

Address correspondence to M. Todd Greene, PhD, MPH, Epidemiologist \& Research Investigator, Ann Arbor VA Health System, University of Michigan Health System Division of General Medicine, Program Manager, VA/UM Patient Safety Enhancement Program, 2800 Plymouth Rd., NCRC Building 16, Room 470C, Ann Arbor, MI 48109 (mtgreene@med.umich.edu). Infect Control Hosp Epidemiol 2016;37:1122-1122

(C) 2016 by The Society for Healthcare Epidemiology of America. All rights reserved. 0899-823X/2016/3709-0022. DOI: 10.1017/ice.2016.143

\section{REFERENCES}

1. National Health Saftety Network e-News. 2015;10(4):6. The Centers for Disease Control and Prevention website. http://www. cdc.gov/nhsn/pdfs/newsletters/nhsn-enewsletter_dec-2015_final.pdf. Published 2015. Accessed June 3, 2016.

2. Fakih MG, Gould CV, Trautner BW, et al. Beyond infection: device utilization ratio as a performance measure for urinary catheter harm. Infect Control Hosp Epidemiol 2016;37:327-333.

\section{Accounting for Competing Events in Multivariate Analyses of Hospital-Acquired Infection Risk Factors}

To the Editor-We congratulate Brown et $\mathrm{al}^{1}$ for the excellent review article about the necessary issues that need to be addressed in multivariate analyses of hospital-acquired infection (HAI) risk factors. We agree that 4 statistical issues should 